05,11

\title{
Влияние внешних воздействий на магнетизм флуктуирующих низкоразмерных электронных и спиновых корреляций в фрустрированных манганитах $\operatorname{La}_{1-y} \mathrm{Sm}_{y} \mathrm{MnO}_{3+\delta}(y=0.85,1.0)$
}

\author{
(C) Ф.Н. Буханько, А.Ф. Буханько
}

Донецкий фризико-технический институт им. А.А. Галкина НАНУ, Киев, Украина

E-mail: metatem@ukr.net

Поступила в Редакцию 8 июля 2019 г.

В окончательной редакции 8 июля 2019 г.

Принята к публикации 15 июля 2019 г.

Исследовано влияние внешних воздействий на температурные зависимости намагниченности фрустрированных манганитов $\mathrm{La}_{1-y} \mathrm{Sm}_{y} \mathrm{MnO}_{3+\delta}(\delta \sim 0.1, y=0.85,1.0)$. Обнаруженные в обоих образцах два острых пика $M(T)$ различной интенсивности при близких температурах $T_{1}$ и $T_{2}$ чуть выше критической температуры $T_{c}$ фазового перехода в когерентное сверхпроводящее состояние соответствуют расходимости Линдхарда $\chi_{L}\left(q_{\text {nest }}\right)$ температурной зависимости парамагнитной восприимчивости страйп-подобных 1D электрон/спиновых корреляций модулированных с волновыми векторами $q_{\text {nest } 1}=2 k_{F 1}$ в и $q_{\text {nest } 2}=2 k_{F 2}$. Появление и эволюция особенностей намагниченности с ростом поля объяснены формированием в $a b$ плоскостях при полном нестинге электрон-дырочных участков поверхности Ферми пространственной модуляции электронных и магнитных свойств в виде фрагментов двух флуктуирующих квазиодномерных волн зарядовой/спиновой плотности несоразмерных с кристаллической решеткой с волновыми векторами $\mathbf{q}_{1} \| a$ и $\mathbf{q}_{2} \| b$ направлениям. Предполагается, что сильная зависимость намагниченности флуктуирующих 1D ВЗП/ВСП корреляций от внешних воздействий вызвана непосредственной близостью свойств образцов к квантовой критической точке.

Ключевые слова: фрустрированные манганиты, квантовый беспорядок, волны плотности состояний, нестинг поверхности Ферми, низкоразмерные корреляции.

DOI: $10.21883 /$ FTT.2019.12.48584.545

\section{1. Введение}

Ранее было установлено, что с ростом уровня допирования купратов на границе между фрустрированной неелевской АФМ и зарождающейся сверхпроводящей (СП) фазой возникает неоднородное страйп-подобное состояние, ответственное по мнению многих авторов [16] за появление СП в купратах. Обычно страйпами называют 1D волны плотности состояний, которые соответствуют модуляции только заряда (зарядовые страйпы) или модуляции сосуществующих состояний $1 \mathrm{D}$ зарядового и спинового порядка (спиновые страйпы). Зарядовые страйпы нарушают симметрию вращения и трансляционную симметрию перпендикулярную страйпам. Параметром порядка $\left\langle\rho_{\mathbf{Q}_{\mathrm{ch}}}\right\rangle$ этого состояния служит Фурье компонента волны зарядовой плотности (ВЗП) с волновым вектором $\mathbf{k}= \pm \mathbf{Q}_{\mathbf{c h}}$. Спиновые страйпы разрушают инвариантность вращения спинов и обращения времени. Параметром порядка $\left\langle\mathbf{s}_{\mathbf{Q}_{s}}\right\rangle$ этого состояния служит Фурье компонента волны спиновой плотности (ВСП) с волновым вектором $\mathbf{k}=\mathbf{Q}_{\mathbf{s}}=\mathbf{Q}_{\mathrm{AF}} \pm 1 / 2 \mathbf{Q}_{\mathbf{c h}}$. В случае формирования спиновых страйпов, обязательно возникают и зарядовые страйпы. ВЗП состояния обычно возникают в пределе слабой связи, если существуют участки Ферми поверхности (ФП) с хорошим нестингом.
В рамках модели сильной связи страйпы соответствуют разделению на микрофазы, вызванному снижением кинетической энергии допированных дырок. В купратах статический страйповый порядок конкурирует со сверхпроводимостью. Критическая температура перехода в ВТСП состояние и энергия конденсации сильно подавляются в материалах, имеющих статический страйповый порядок. Однако медлено флуктуирующий во времени страйповый порядок, согласно существующим моделям, может сосуществовать со стабильной сверхпроводимостью и быть включенным в механизм спаривания.

В работе [6] была исследована проблема экспериментального обнаружения флуктуирующих зарядовых страйпов в фазе с квантовым беспорядком. В этой фазе отсутствует состояние с дальним страйповым порядком при $T \rightarrow 0$, но имеются другие виды дальнего порядка, например, СП порядок. Динамический структурный фактор $S_{\mathrm{ch}}(\mathbf{k}, \omega)$ флуктуирующей ВЗП для значений векторов $\mathbf{k}$ вблизи $\mathbf{Q}_{\mathbf{c h}}$ измеряет коллективные флуктуации, которые наиболее чувствительны к положению квантовой критической точки (QCP). Особый интерес представляет флуктуирующий порядок в страйпах в непосредственной близости к квантовой критической точке. Была рассмотрена фаза с квантовым беспорядком в непосредственной близости к QСР. Вне этой области 
основное состояние предполагается упорядоченным в виде зарядовой страйповой фазы. По мере приближения к QCP происходит расхождение корреляционной длины и времени скейлинга. В квантовой разупорядоченной фазе существует $E_{\mathrm{G}}$ щель в спектре флуктуаций. Для энергий флуктуаций $\hbar \omega \geq E_{\mathrm{G}}$ в динамическом структурном факторе $S(\mathbf{k}, \omega)$ имеется полюс, соответствующий резко обозначенному элементарному возбуждению. Эта мягкая мода конденсируется вблизи QСР. Для энергий флуктуаций $\hbar \omega>3 E_{\mathrm{G}}$ структурный фактор проявляет свойства многочастичного континуума.

В классическом пределе динамический структурный фактор тесно связан со статической восприимчивостью простым соотношением $S(\mathbf{k})=T_{\chi}(\mathbf{k}, \omega=0)$. Вблизи квантовой критической точки эта связь значительно сложнее. В отсутствие структурного беспорядка статические измерения не могут чувствовать флуктуирующий порядок. Однако при достаточно высоком беспорядке флуктуации могут оказывать воздействие на низкоэнергетические состояния. Слабый струтурный беспорядок создает низкочастотную квазиупругую часть спектральной функции $S(\mathbf{k}, \omega)$. Другими словами беспорядок исключает из рассмотрения спектральную щель, но мало изменяет интегральную спектральную функцию. В присутствии слабого структурного беспорядка функция $S(\mathbf{k}, \omega=0)$ должна проявлять дисперсию подобную чистой системе и поэтому ее можно использовать для определения природы соседней упорядоченной фазы. Таким образом, статическая магнитная воспримчивость $\chi(\mathrm{k}, T)$ флуктуирующей страйповой фазы связана в классичесом пределе с динамическим структурным фактором $S(\mathbf{k}, \omega=0)$ простым соотношением $\chi(k, T)=S(k) / T$. Это позволяет предположить, что измерения статической магнитной восприимчивости в системах с малым структурным беспорядком можно использовать как источник информации о интегральной спектральной функции флуктуирующих спиновых страйповых фаз. В фазе с квантовым беспорядком наличие любого параметра порядка делает очевидным связь системы с внешним воздействием нарушающим ее симметрию. В случае зарядовых страйпов, фурье компонента $V_{\mathbf{k}}$ слабого потенциала приложенного внешнего поля приводит к линейному отклику $\left\langle\rho_{\mathbf{k}}\right\rangle=\chi_{\mathrm{ch}}(\mathbf{k}) V_{\mathbf{k}}+\ldots$ системы зарядовых страйпов с флуктуирующим квантовым беспорядком. Существует также вопрос о причинах появления волн плотности состояний того или другого типа. Согласно [6], при формировании ВСП отсутствует разница между пределом сильной связи, в котором спиновые страйпы можно рассматривать как фазовое разделение на металлические полосы избыточного заряда и почти непроводящие АФМ полосы между ними, и хартри-фоковским описанием спиновых страйпов в пределе слабой связи. В пределе слабой связи ВСП появляется в результате открытия малых щелей на различных сегментах ФП при хорошем нестинге.

Авторы [6] отмечают, что при наличии нескольких конкурирующих параметров порядка построенная ими фазовая диаграмма ВТСП купратов сильно усложняется. Так например, в простейшем случае нулевого структурного беспорядка на $T-x$ фазовой диаграмме вблизи QСР реализуется режим „флуктуирующие страйпы“, для которого характерен значительный локальный страйповый порядок, управляемый близостью к QСР. Квантовая критическая точка находится вблизи границы, разделяющей фазу с дальним страйповым порядком и сверхпроводящую упорядоченную фазу. Вблизи QСР степень страйпирования на локальном уровне значительно превышает слабую компоненту с дальним порядком. В более сложном случае слабого структурного беспорядка на $T-x$ фазовой диаграмме присутствуют различные страйп-подобные фазы и СП фаза. Могут возникать различные виды $T-x$ фазовых диаграмм в случаях: соразмерного и несоразмерного, диагонального и вертикального, центрированного на связях и на узлах страйпового порядка. Так как СП порядок подавляется в вихревом состоянии, индуцированном внешним магнитным полем $H>G_{c 2}$, то исследование поведения системы во внешнем магнитном поле является лучшим методом изучения взаимодействия страйпового и СП порядка вблизи QCP.

Ранее, нами были обнаружены характерные признаки сосуществования в фрустрированных манганитах при температурах ниже $60 \mathrm{~K}$ нанофаз спиновой, электрон-дырочной и сверхпроводящей квантовых жидкостей $[7,8]$ в нулевом (слабом) внешнем магнитном поле. В данной работе было проведено детальное исследование особенностей намагниченности $M(T)$ образцов $\mathrm{La}_{1-y} \mathrm{Sm}_{y} \mathrm{MnO}_{3+\delta}(\delta \sim 0.1, y=0.85,1.0)$ с квантовым беспорядком в интервале температур $4.2-100 \mathrm{~K}$, измеренных в полях $H=7,100,350,1050$ и 3500 Ое в ZFC-режиме измерений. Сильная зависимость найденных особенностей от небольших изменений концентрации $\mathrm{Sm}$ и напряженности внешнего магнитного поля объяснена ростом флуктуаций нескольких локальных параметров порядка, возникающих в непосредственной близости к квантовой критической точке.

\section{2. Методика эксперимента}

Образцы самодопированных манганитов $\mathrm{La}_{1-y} \mathrm{Sm}_{y} \mathrm{MnO}_{3+\delta}(\delta \sim 0.1, y=0.85,1.0)$ были получены из высокочистых окислов лантана, самария и электролитического марганца, взятых в стехиометрическом соотношении. Синтезированный порошок прессовали под давлением $10 \mathrm{kbar}$ в диски диаметром $6 \mathrm{~mm}$, толщиной $1.2 \mathrm{~mm}$ и спекали на воздухе при температуре $1170^{\circ} \mathrm{C}$ в течение $20 \mathrm{~h}$ с последующим снижением температуры со скоростью $70^{\circ} \mathrm{C} / \mathrm{h}$. Полученные таблетки представляли собой однофазную по рентгеновским данным керамику. Рентгенографические исследования проводили при $300 \mathrm{~K}$ на дифрактометре ДРОН-1.5 в излучении $\mathrm{NiK}_{\alpha 1+\alpha 2}$. Симметрия и параметры кристаллической решетки определялись по положению и 
характеру расщепления рефлексов псевдокубической решетки типа перовскита. Измерения температурных зависимостей намагниченности образцов производились на вибрационном магнитометре VSM EGG, Princeton Aplied Reserch в интервале температур 4.2-250 K и непромышленном магнитометре в постоянных магнитных полях $7 \mathrm{Oe}, 100 \mathrm{Oe}, 350 \mathrm{Oe}, 1050 \mathrm{Oe}$ и 3500 Oе в ZFC-режиме измерений.

\section{3. Экспериментальные результаты и их обсуждение}

Согласно рис. 1, в образце $\mathrm{La}_{0.15} \mathrm{Sm}_{0.85} \mathrm{MnO}_{3+\delta}$ в слабом внешнем магнитном поле $H=7$ Ое при температурах ниже $60 \mathrm{~K}$ реализуется диэлектрическое немагнитное состояние типа квантовой спиновой жидкости (КСЖ), формирование которой было впервые обнаружено нами раннее в системе $\mathrm{La}_{1-y} \mathrm{Sm}_{y} \mathrm{MnO}_{3+\delta}$ с ростом концентрации $\mathrm{Sm}$ [7]. Температурная зависимость намагниченности имеет вид слабого широкого пика намагниченности с вершиной вблизи $20 \mathrm{~K}$, размазанного в широком интервале температур $4.2-65 \mathrm{~K}$. При температурах $T \rightarrow 0$ наблюдается экспоненциальное падение намагниченности образца к нулю, что характерно для щелевой квантовой спиновой жидкости. Слабый рост напряженности магнитного поля до $100 \mathrm{Oe}$ привел к радикальным изменениям температурной зависимости намагниченности. Как видно из рис. 2, с ростом $H$ появилась пик-холм особенность намагниченности $\mathrm{La}_{0.15} \mathrm{Sm}_{0.85} \mathrm{MnO}_{3+\delta}$ в виде дублета острых пиков намагниченности с существенно различной интенсивностью при близких температурах $T_{1} \cong 42 \mathrm{~K}$ и $T_{2} \cong 58 \mathrm{~K}$.

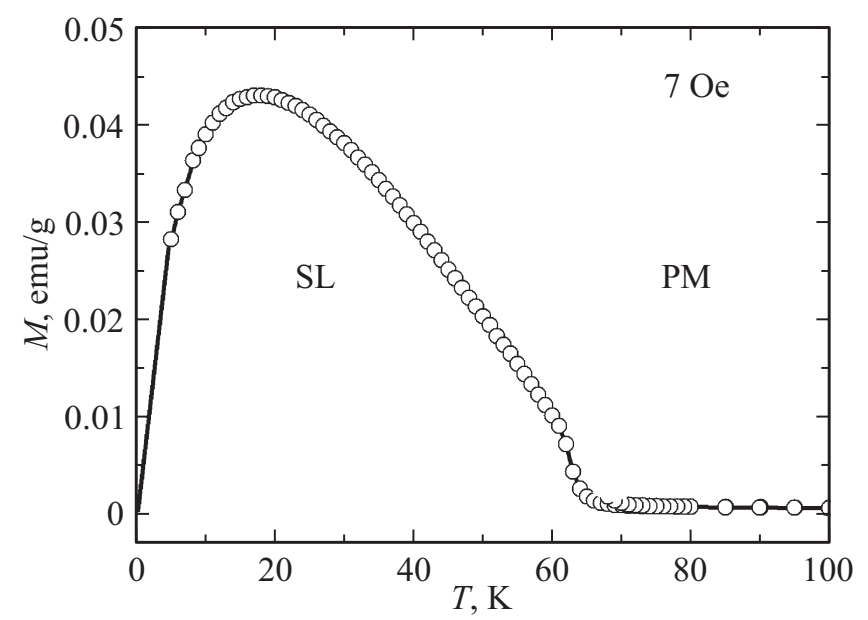

Рис. 1. Широкий слабый пик температурной зависимости намагниченности $\mathrm{La}_{0.15} \mathrm{Sm}_{0.85} \mathrm{MnO}_{3+\delta}$, размазанный в интервале температур $0 \leq T \leq 70 \mathrm{~K}$ вблизи критической температуры $\sim 20 \mathrm{~K}$ фазового перехода системы одномерных электрон/спиновых корреляций в неупорядоченное состояние типа щелевой квантовой спиновой жидкости с резким (экспоненциальным) падением намагниченности к нулю при $T \rightarrow 0$ (поле $H=7 \mathrm{Oe})$.

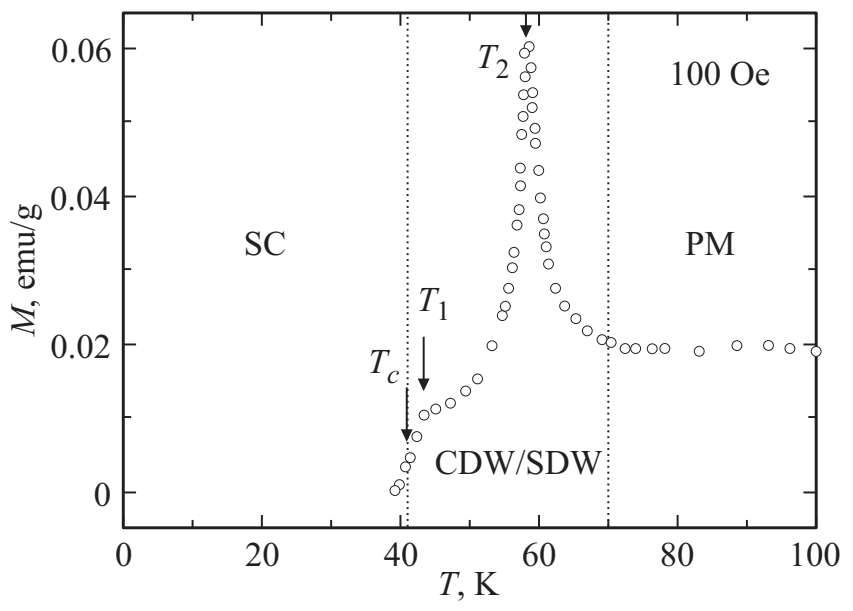

Рис. 2. Пик-холм особенность температурной зависимости намагниченности $\mathrm{La}_{0.15} \mathrm{Sm}_{0.85} \mathrm{MnO}_{3+\delta}$ в поле $H=100 \mathrm{Oе}$ в виде дублета острых пиков с существенно различной интенсивностью при близких температурах $T_{1} \cong 42 \mathrm{~K}$ и $T_{2} \cong 58 \mathrm{~K}$ чуть выше критической температуры $T_{c} \cong 41 \mathrm{~K}$ фазового перехода в когерентное сверхпроводящее состояние. Острые пики намагниченности соответствуют расходимости Линдхарда температурной зависимости парамагнитной восприимчивости $\chi_{L}\left(q_{\text {nest }}\right)$ системы 1D электрон/спиновых корреляций при значениях волнового вектора $q_{n e s t 1}=2 k_{F 1}\left(\mathbf{q}_{1} \| a\right)$ и $q_{n e s t 2}=2 k_{F 2}\left(\mathbf{q}_{1} \| b\right)$ с большой и малой плотностью состояний на уровне Ферми $N_{1}\left(E_{\mathrm{F}}\right) \ll N_{2}\left(E_{\mathrm{F}}\right)$.

Найденая впервые в допированных манганитах особенность $M(T)$ в виде двух пиков намагниченности накладывается на резкое падение температурной зависимости намагниченности до нуля при дальнейшем понижении температуры до критического значения $T_{c} \cong 41 \mathrm{~K}$ фазового перехода в СП состояние. Появление пик-холм особенности намагниченности при температурах чуть выше $T_{c}$ мы связываем с формированием в образцах в полях $H \geq 100$ Ое фрагментов связанных волн зарядовой и спиновой плотности (ВЗП/ВСП) несоразмерных с кристаллической решеткой. Предполагается, что обнаруженные в поле $H=100$ Ое два острых пика $M(T)$ различной интенсивности при близких температурах $T_{1}$ и $T_{2}$ чуть выше критической температуры $T_{c} \cong 41 \mathrm{~K}$ фазового перехода в локальное сверхпроводящее состояние, соответствуют хорошо известной в литературе [915] расходимости Линдхарда температурной зависимости парамагнитной восприимчивости 1D газа электронов $\chi_{L}\left(q_{\text {nest }}\right)$ на $E_{\mathrm{F}}$ при значениях волнового вектора $q_{n e s t 1}=2 k_{F 1}$ в и $q_{n e s t 2}=2 k_{F 2}$ в двух пространственно разделенных областях образца (условно „металлическая“ и ,диэлектрическая“" фаза) с большой и малой плотностью состояний свободных носителей на уровне Ферми $N 1\left(E_{\mathrm{F}}\right) \ll N_{2}\left(E_{\mathrm{F}}\right)$.

Появление таких областей в $\mathrm{La}_{0.15} \mathrm{Sm}_{0.85} \mathrm{MnO}_{3+\delta}$ можно объяснить формированием в $a b$ плоскостях $\mathrm{La}_{0.15} \mathrm{Sm}_{0.85} \mathrm{MnO}_{3+\delta}$ при температурах $T<60 \mathrm{~K}$ пространственной модуляции электронных и магнитных 
свойств в виде фрагментов флуктуирующих квазиодномерных волн зарядовой/спиновой плотности несоразмерных с кристаллической решеткой с волновыми векторами $\mathbf{q}_{1} \| a$ и $\mathbf{q}_{2} \| b$ направлениям. Это приводит к фазовому разделению образца на квазиодномерные „металлические“ ФМ и „диэлектрические“ АФМ нанофазы, сосуществующие в динамическом режиме в виде наномасштабных квазиодномерных ферромагнитных и антиферромагнитных спиновых корреляций. Повидимому, сосуществование в слабых магнитных полях ортогональных 1D электронных и спиновых корреляций приводит к появлению двумерных сверхпроводящих контуров ответственных за локальную сверхпроводимость в фрустрированных манганитах, подобных обнаруженным ранее в ВТСП купратах. Результаты проведенных в работе [8] исследований аномалий температурных и полевых зависимостей $\mathrm{dc}$ намагниченности образцов $\mathrm{La}_{1-y} \mathrm{Sm}_{y} \mathrm{MnO}_{3+\delta}$ с концентрацией самария $y=0.85$ и 1.0 в интервале температур $4.2-100 \mathrm{~K}$ свидетельствуют о существовании в этих образцах когерентных джозефсоновских наноструктур в виде СП контуров со средним линейным размером $\langle a\rangle \sim 1.4 \cdot 10^{2} \mathrm{Hм}$.

В работе [8] было также показано, что присутствие подобных джозефсоновских наноструктур (микродоменов с размерами порядка длины когерентности) было обнаружено ранее во многих ВТСП купратах и имеет различную природу. Так например, по данным измерения сопротивления и намагниченности были обнаружены когерентные джозефсоновские наноструктуры в $\mathrm{Y}_{1} \mathrm{Ba}_{2} \mathrm{Cu}_{3} \mathrm{O}_{7-\delta}$ тонких пленках с высоким критическим током. Наноструктуры были обнаружены ранее и в монокристаллах $\mathrm{Y}_{1} \mathrm{Ba}_{2} \mathrm{Cu}_{3} \mathrm{O}_{7-\delta}(\delta<0.1)$ при исследовании структуры кристаллической решетки при $300 \mathrm{~K}$ с помощью электронной микроскопии высокого разрешения. Помимо хорошо известных границ двойникования расположенных на расстоянии $\sim 500 \AA$, было обнаружено существование в $a b$-плоскостях наноячеек с линейными размерами $\sim 10-20 \AA$, связанных джозефсоновскими туннельными переходами. Наноячейки формируют неравномерную сетку со стенками ячеек ориентированными вдоль направлений, составляющим угол $45^{\circ}$ по отношению к осям (100) и (010). Появление наноструктуры автор связывает с локальным возмущением распределения зарядовой плотности в $a b$-плоскостях. Это, повидимому, универсальное свойство для ҮВСО $(\delta<0.1)$ образцов, приготовленных в различных условиях. Наноячейки связываются в сетку слегка разориентированных доменов, чтобы уменьшить внутренние напряжения. Грубый порядок наноячеек в каждом исследованном кристалле подразумевает воздействие полей дальнодействующих деформаций кристаллической решетки. Двумерные сетки наноячеек скоррелированы вдоль $c$-оси. Эти экспериментальные результаты поддерживают дискретные теоретические модели, предложенные для объяснения различных физических свойств высокотемпературных сверхпроводников, таких как: теплоемкость в магнитном поле, сопротивление магнитного потока, фа-

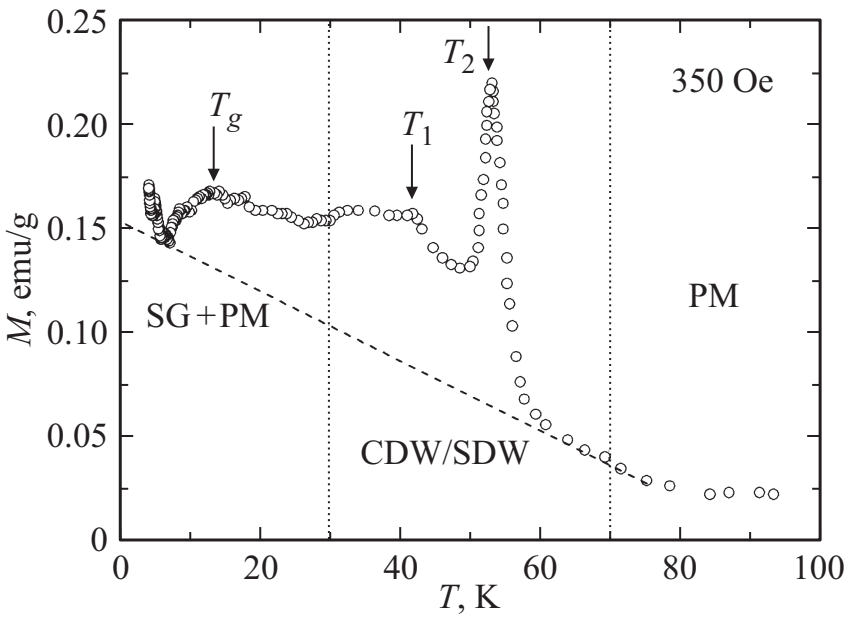

Рис. 3. Пик-холм особенность температурной зависимости намагниченности $\mathrm{La}_{0.15} \mathrm{Sm}_{0.85} \mathrm{MnO}_{3+\delta}$ в поле $H=350 \mathrm{Oe}$ в виде дублета острых пиков с существенно различной интенсивностью при близких температурах $T_{1} \cong 42 \mathrm{~K}$ и $T_{2} \cong 52 \mathrm{~K}$ накладывается на дополнительный положительный вклад 1D электрон/спиновых корреляций в суммарную намагниченность, практически линейно растущий с понижением температуры от $80 \mathrm{~K}$ до $4.2 \mathrm{~K}$. С ростом поля от нуля до 350 Ое появляется слабая пиковая особенность $M(T)$, связанная с фазовым переходом 1D электрон/спиновых корреляций в неупорядоченное „замороженное“ состояние типа спинового стекла с температурой $T_{G} \cong 12 \mathrm{~K}$.

зовые переходы в сверхпроводнике с колонообразными дефектами, эффект флуктуаций фазы. В каждом случае использовалось дискретное представление, в котором сверхпроводящие слои были описаны как построения из очень маленьких „гранул“ с размерами сравнимыми с длиной когерентности и связанных вместе джозефсоновскими переходами. Результаты экспериментальных и теоретических исследований позволили предположить, что джозефсоновские наноструктуры в $a b$-плоскостях YВСО должны действовать на все физические свойства этого высокотемпературного сверхпроводника. Следует отметить, что причины формирования и свойства сверхпроводящих наноструктур в купратах и манганитах различны.

Дальнейший рост напряженности магнитного поля в $\mathrm{La}_{0.15} \mathrm{Sm}_{0.85} \mathrm{MnO}_{3+\delta}$ до $350 \mathrm{Oe}$ не привел к существенным изменениям пик-холм особенности намагниченности образца. Вместе с тем, исчез отрицательный вклад локальной сверхпроводимости в суммарную намагниченность и появился дополнительный положительный вклад практически линейно растущий с понижением температуры от $80 \mathrm{~K}$ до $4.2 \mathrm{~K}$ (рис. 3). Дополнительный вклад в намагниченность обусловлен, по-видимому, смещением широкого пика плотности состояний квазичастиц с энергией меньшей $E_{\mathrm{F}}$ и тесно связан с уменьшением псевдощели в непрерывном спектре элементарных носителей заряда/спина вблизи $E_{\mathrm{F}}$, вызванным ростом напряженности внешнего магнитного поля. 
Подобная „металлизация“ спектра квазичастиц в результате роста уровня допирования свободными дырками наблюдалась ранее в двухслойных манганитах [9-15]. Более того, в магнитном поле 350 Ое на дополнительный квазилинейный рост намагниченности $M(T)$ с понижением температуры до $4.2 \mathrm{~K}$ накладывается пиковая особенность вблизи $12 \mathrm{~K}$, которую мы связываем с фазовым переходом газа электронов в неупорядоченное состояние их спинов типа спинового стекла с температурой „замерзания“ спинов $T_{G} \cong 12 \mathrm{~K}$, индуцированный слабым ростом внешнего магнитного поля. Резкий рост намагниченности при температурах ниже $7 \mathrm{~K}$, по-видимому, обусловлен формированием в поле 350 Ое кластеров неупорядоченных спинов квазичастиц, которые вносят существенный „суперпарамагнитный“ кюри-подобный вклад в намагниченность образцов при температурах близких к $T=0$.

Намагниченность образца с $y=0.85$ кардинально изменилась при измерениях в магнитном поле $H=1050$ Oе (рис. 4). Во-первых, произошло сильное уширение пиков при $T_{1}$ и $T_{2}$ и выравнивание их интенсивностей. Во-вторых, вместо пиковой особенности вблизи $12 \mathrm{~K}$ возникла пороговая особенность вблизи критической температуры $T_{\mathrm{FM}} \approx 12 \mathrm{~K}$, характерная для фазового перехода в упорядоченную ФМ фазу. Квазилинейный вклад в намагниченность в интервале температур 4.2-100 K существенно вырос. Завершающим этапом эволюции температурных зависимостей намагниченности $M(T)$ в $\mathrm{La}_{0.15} \mathrm{Sm}_{0.85} \mathrm{MnO}_{3+\delta}$ с ростом напряженности внешнего магнитного поля можно считать результаты измерений в поле $H=3.5 \mathrm{kOe}$, представлен-

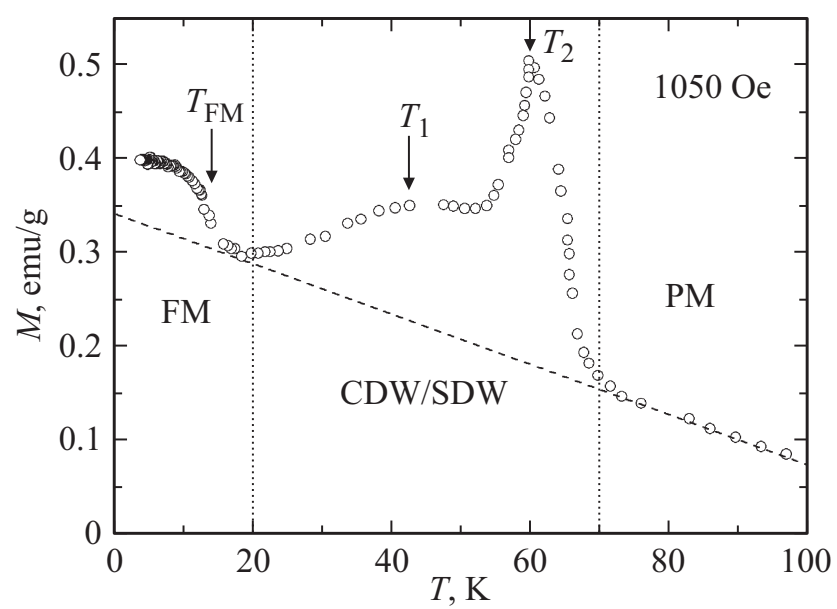

Рис. 4. Изменения температурной зависимости намагниченности $\mathrm{La}_{0.15} \mathrm{Sm}_{0.85} \mathrm{MnO}_{3+\delta}$ в поле $H=1050$ Oе: 1) сильное уширение дублета пиков при температурах $T_{1} \cong 42 \mathrm{~K}, T_{2} \cong 60 \mathrm{~K}$ и выравнивание их интегральных интенсивностей; 2) с ростом поля возникла пороговая особенность вблизи температуры $T_{F M} \cong 12 \mathrm{~K}$, характерная для фазового перехода в ферромагнитную микрофазу; 3) квазилинейный положительный вклад низкоразмерных электрон/спиновых корреляций в суммарную намагниченность в интервале температур $4.2-100 \mathrm{~K}$ существенно вырос.

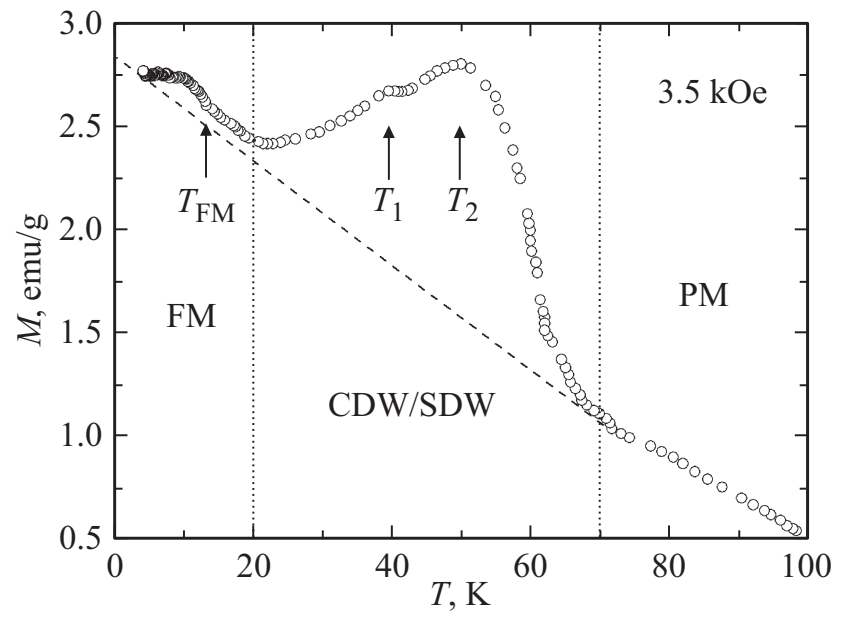

Рис. 5. Изменения температурной зависимости намагниченности $\mathrm{La}_{0.15} \mathrm{Sm}_{0.85} \mathrm{MnO}_{3+\delta} \quad$ в поле $H=3500 \mathrm{Oe}$ : 1) низкотемпературный пик намагниченности вблизи температуры $T_{1}=42 \mathrm{~K}$ с ростом поля практически исчезает; 2) доминирующий вклад в намагниченность вносит широкий пик с вершиной вблизи температуры $T_{2}=50 \mathrm{~K}$, размазанный в интервале температур $20-70 \mathrm{~K} ; 3$ ) сильный рост величины положительного квазилинейного вклада низкоразмерных электрон/спиновых корреляций в суммарную намагниченность в интервале температур $4.2-100 \mathrm{~K}$.

ные на рис. 5. Согласно рис. 5, низкотемпературный пик намагниченности вблизи температуры $T_{1}=40 \mathrm{~K}$ практически исчезает. Доминирующий вклад в намагниченность образца вносит широкий пик с вершиной вблизи температуры $T_{2}=50 \mathrm{~K}$, размазанный в интервале температур $20-70 \mathrm{~K}$. Следует отметить также рост величины квазилинейного вклада в намагниченность в интервале температур $4.2-100 \mathrm{~K}$ и сохранение пороговой особенности вблизи температуры $T_{\mathrm{FM}} \approx 12 \mathrm{~K}$. Таким образом, рост напряженности $H$ внешнего магнитного поля в интервале полей $350 \mathrm{Oe} \leq H \leq 3500$ Ое приводит к росту плотности состояний свободных носителей заряда/спина на $E_{\mathrm{F}}$, что означает подавление зарядовой псевдощели в спектре квазичастиц магнитным полем, то есть „металлизацию“ 1D В3П/ВСП корреляций, а также появление аномального ферромагнетизма при температурах ниже $12 \mathrm{~K}$. Найденное нами сильное уширение пиковых особенностей вблизи $T_{2}$ с ростом магнитного поля (рис. 4,5) можно объяснить, в рамках рассмотренных выше моделей, появлением сильной связи газа одноэлектронных носителей заряда/спина на $E_{\mathrm{F}}$ с коллективными возбуждениями ( магнонами), что приводит к перенормировке их спектра по сравнению с параболической дисперсией характерной для обычных металлов.

Как видно из рис. 6, в образце $\mathrm{SmMnO}_{3+\delta}$ в слабом внешнем магнитном поле $H=7$ Ое при температурах ниже $60 \mathrm{~K}$ также реализуется диэлектрическое немагнитное состояние типа щелевой квантовой спиновой жидкости. Полученная в магнитном поле $H=7 \mathrm{Oe}$ в 
ZFC-режиме измерений температурная зависимость намагниченности $\mathrm{SmMnO}_{3+\delta}$, имеет вид слабого широкого пика намагниченности $M(T)$ с вершиной вблизи $20 \mathrm{~K}$, размазанного в интервале температур 4.2-60K. При температурах $T \rightarrow 0$ наблюдается экспоненциальное падение намагниченности образца к нулю, характерное для щелевой квантовой спиновой жидкости. С ростом магнитного поля до 100 Ое температурная зависимость

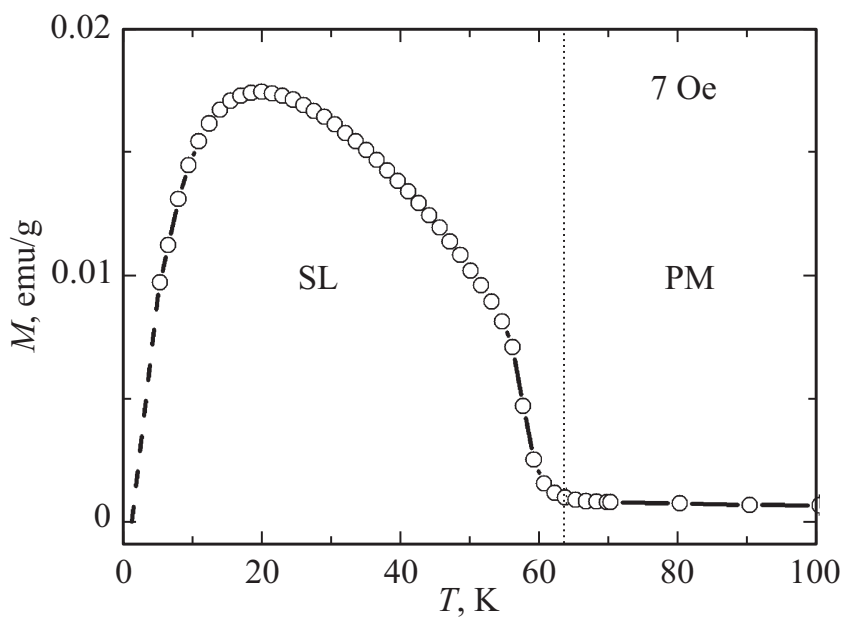

Рис. 6. Широкий слабый пик температурной зависимости намагниченности $\mathrm{SmMnO}_{3+\delta}$, размазанный в интервале температур $0 \leq T \leq 70 \mathrm{~K} \mathrm{~K}$ вблизи критической температуры $\sim 20 \mathrm{~K}$ фазового перехода одномерных электрон/спиновых корреляций в неупорядоченное состояние типа щелевой квантовой спиновой жидкости с резким (экспоненциальным) падением намагниченности к нулю при $T \rightarrow 0$ (поле $H=7 \mathrm{Oe}$ ).

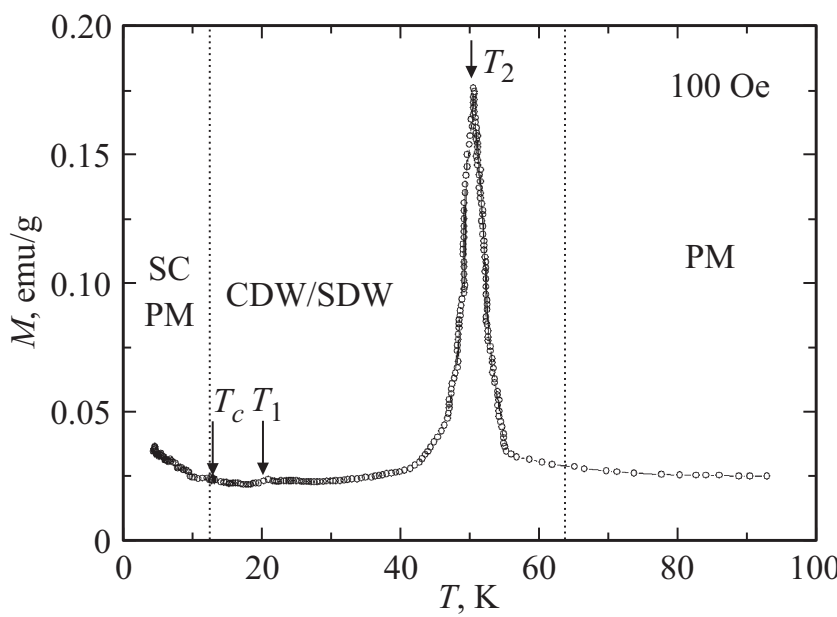

Pис. 7. Интенсивный острый пик температурной зависимости намагниченности $\mathrm{SmMnO}_{3+\delta}$, в поле $H=100$ Ое вблизи температуры $T_{2} \cong 50 \mathrm{~K}$, вызванный расходимостью парамагнитной восприимчивости электрон/спиновых корреляций $\chi_{L}\left(q_{\text {nest } 2}\right)$. Острый пик намагниченности вблизи $T_{1} \cong 20 \mathrm{~K}$ выше критической температуры $T_{c} \cong 12 \mathrm{~K}$ фазового перехода $\mathrm{SmMnO}_{3+\delta}$ в когерентное сверхпроводящее состояние ничтожно мал, но конечен. При $T \rightarrow 0$ намагниченность $\mathrm{SmMnO}_{3+\delta}$ расходится по закону Кюри.

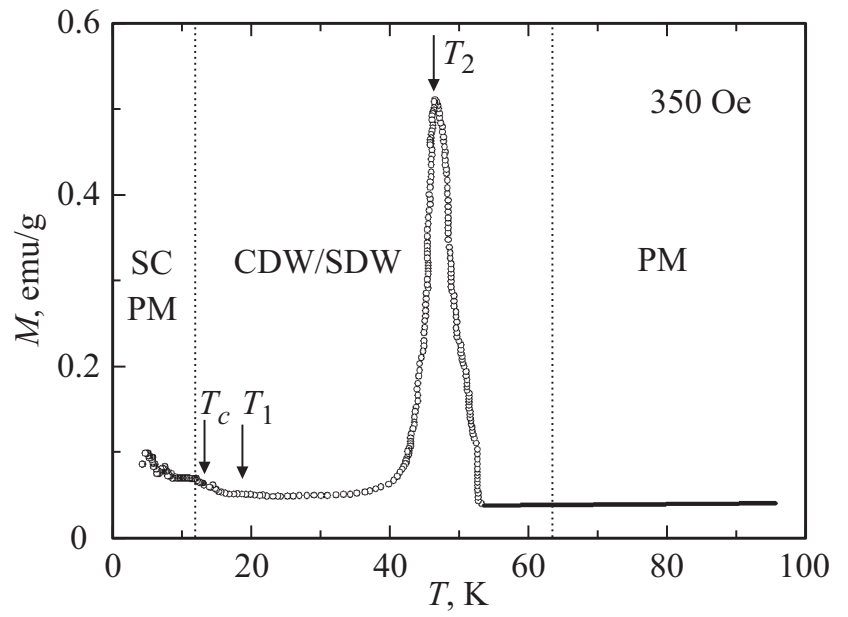

Рис. 8. Интенсивный острый пик температурной зависимости намагниченности $\mathrm{SmMnO}_{3+\delta}$, в поле $H=350$ Ое вблизи температуры $T_{2} \cong 50 \mathrm{~K}$, вызванный расходимостью парамагнитной восприимчивости электрон/спиновых корреляций $\chi_{L}\left(q_{\text {nest } 2}\right)$. Острый пик намагниченности вблизи $T_{1} \cong 20 \mathrm{~K}$ выше критической температуры $T_{c} \cong 12 \mathrm{~K}$ фазового перехода $\mathrm{SmMnO}_{3+\delta}$ в когерентное сверхпроводящее состояние ничтожно мал, но конечен. При $T \rightarrow 0$ намагниченность $\mathrm{SmMnO}_{3+\delta}$ расходится по закону Кюри.

намагниченности резко изменилась. Как видно из рис. 7 , при температурах $T_{1}=20 \mathrm{~K}$ и $T_{2}=50 \mathrm{~K}$ чуть выше $T_{c}=12 \mathrm{~K}$ появились пиковые особенности намагниченности $M(T)$, подобные рассмотренным нами выше. В отличие от образца с $y=0.85$, в $\mathrm{SmMnO}_{3+\delta}$ интенсивность пиковой особенности вблизи температуры $T_{1}$ ничтожно мала, но конечна. Следует также отметить небольшой кюри-подобный рост намагниченности при $T \rightarrow 0$. Увеличение напряженности измерительного поля до 350 Ое не привело к качественному изменению температурной зависимости намагниченности (рис. 8). Кардинальные изменения намагниченности $M(T)$ были обнаружены при измерениях в магнитном поле $H=1 \mathrm{kOe}$. Как видно из рис. 9, рост магнитного поля привел к уменьшению скачка Костерлитца-Таулесса намагниченности образца вблизи температуры $T_{c}=12 \mathrm{~K}$ его перехода в когерентное СП состояние, полному подавлению пика намагниченности вблизи $T_{1}$ и значительному уширению пика вблизи $T_{2}$. Завершающим этапом эволюции температурных зависимостей намагниченности $M(T)$ в $\mathrm{SmMnO}_{3+\delta}$ с ростом напряженности внешнего магнитного поля можно считать результаты измерений в поле $H=3.5 \mathrm{kOe}$, представленные на рис. 10. При температурах ниже $60 \mathrm{~K}$ перехода образца из парамагнитной фазы в фазу с периодическим наномасштабным упорядочением квазичастиц в виде флуктуирующих ВЗП/ВСП фрагментов сохраняется лишь широкий пик намагниченности вблизи температуры $T_{2} \approx 45 \mathrm{~K}$. В результате роста напряженности внешнего магнитного поля в интервале полей $7 \mathrm{Oe} \leq H \leq 3500$ Ое в образце $\mathrm{SmMnO}_{3+\delta}$ сформировалось при температурах ниже 


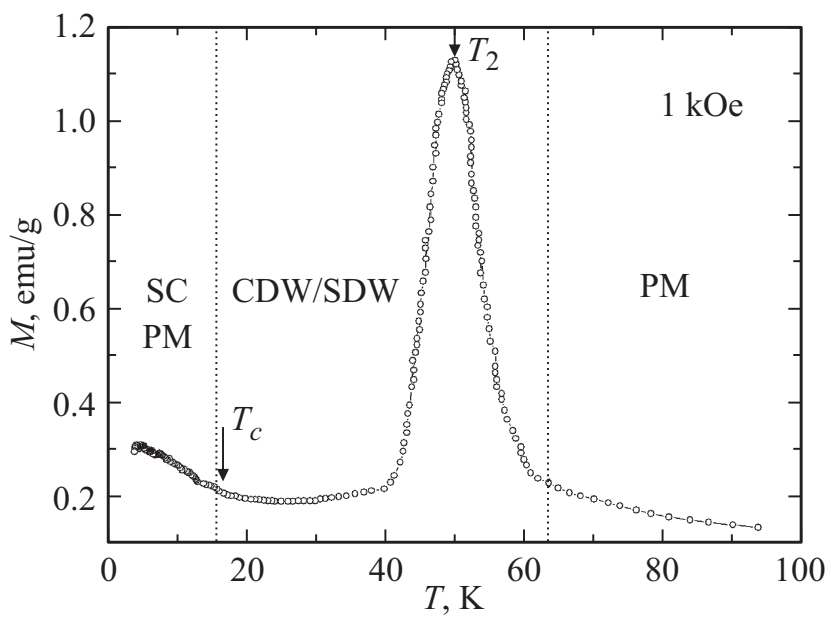

Рис. 9. Уширение интенсивного пика температурной зависимости намагниченности $\mathrm{SmMnO}_{3+\delta}$, в поле $H=1050 \mathrm{Oe}$ вблизи температуры $T_{2} \cong 50 \mathrm{~K}$, вызванного расходимостью парамагнитной восприимчивости $\chi_{L}\left(q_{\text {nest } 2}\right)$ электрон/спиновых корреляций вдоль $b$-оси. Полное подавление острого пика намагниченности вблизи температуры $T_{1} \cong 20 \mathrm{~K}$, вызванного расходимостью парамагнитной восприимчивости $\chi_{L}\left(q_{n e s t 1}\right)$ электрон/спиновых корреляций вдоль $a$-оси. При $T \rightarrow 0$ намагниченность $\mathrm{SmMnO}_{3+\delta}$ расходится по закону Кюри.

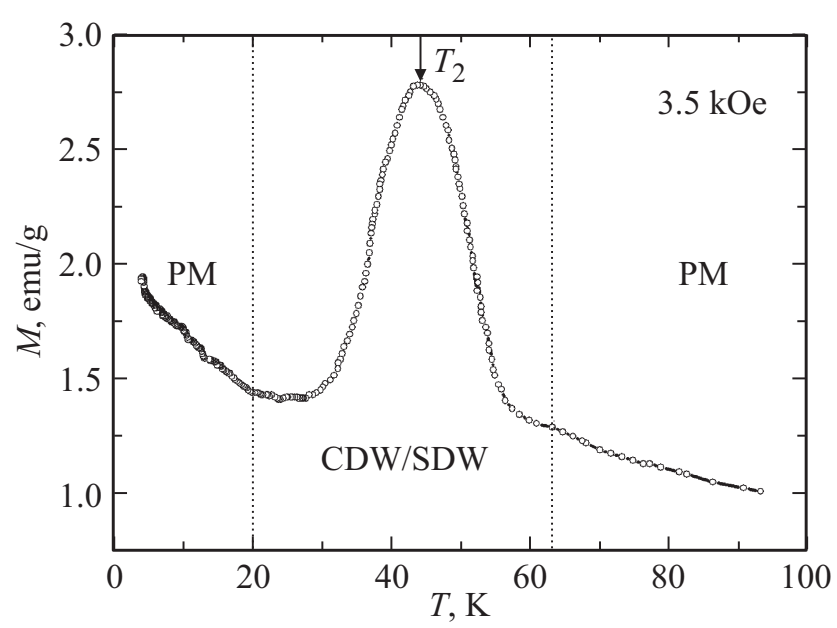

Рис. 10. Сильное уширение интенсивного пика температурной зависимости намагниченности $\mathrm{SmMnO}_{3+\delta}$, в поле $T=3500$ Ое вблизи температуры $T_{2} \cong 45 \mathrm{~K}$, вызванного расходимостью парамагнитной восприимчивости $\chi_{L}\left(q_{\text {nest2 }}\right)$ электрон/спиновых корреляций вдоль $b$-оси. При $T \rightarrow 0$ намагниченность $\mathrm{SmMnO}_{3+\delta}$ расходится по закону Кюри.

$60 \mathrm{~K}$ „металлическое“ состояние типа ВСП с довольно высокой плотностью свободных квазичастиц на уровне Ферми. Это свидетельствует о малой величине (отсутствии) зарядовой псевдощели в электронном спектре носителей заряда/спина в $\mathrm{SmMnO}_{3+\delta}$ на $E_{\mathrm{F}}$ в магнитном поле $H=3.5 \mathrm{kOe}$. Таким образом, рост напряженности поля до значения $H=3.5 \mathrm{kOe}$ привел к подавлению зарядовой псевдощели в спектре квазичастиц, к умень- шению амплитуды ВЗП и, соответственно, к росту амплитуды флуктуирующей 1D BCП несоразмерной с кристаллической решетки. Рост ширины пика $M(T)$ вблизи $T_{2}$ с ростом $H$ вызван, по-видимому, перенормировкой спектра связью с магнонами.

Наиболее детально формирование ВЗП/ВСП состояний в слоистых допированных манганитах, вызванных нестингом ФП и появления при этом пиковой особенности Линдхарта парамагнитной восприимчивости 1D электронного газа, были исследованы экспериментально методом фотоэмиссионой спектроскопии с высоким угловым разрешением (ARPES) [9-15]. Согласно этим работам, при наличии нестинга электрон-дырочных участков поверхности Ферми внешнее воздействие (допинг, давление, магнитное поле) может существенно повлиять на возникновение ВЗП/ВСП и псевдощель в квазидвумерных манганитах за счет изменения геометрии ФП. В работе [10] было получено, что зависимость спектрального веса эмитированных электронов от энергии связи $E$ относительно уровня $E_{\mathrm{F}}$ имеет широкий максимум вокруг $E \sim 1 \mathrm{eV}$. C ростом угла эмиссии широкий пик смещается в сторону $E_{\mathrm{F}}$, уменьшается по интенсивности и практически не наблюдаем для энергии связи $<0.3 \mathrm{eV}$. Этот результат был объяснен существованием вблизи $E_{\mathrm{F}}$ псевдощели в спектре носителей заряда, в которой теряется 90\% спектрального веса. Предполагается, что причиной образования псевдощели в спектре дырок являются плоские участки ФП, которые способствуют появлению всякого рода неустойчивостей связанных с нестингом, что характерно для квазиодномерных систем. Это предположение подтверждается хорошо известным в литературе формированием в этих соединениях квазиодномерных структур (страйпов) с пространственной модуляцией электронных, магнитных и структурных свойств. Связь плоских участков поверхности Ферми осуществляется при определенных значениях волнового вектора $k_{\text {nest }}$ и сопровождается появлением диэлектрической щели $\Delta$ в спектре носителей заряда, a также периодической деформацией кристаллической решетки вызванной нестингом ФП. Для одномерного электронного газа была найдена функция отклика газа $\chi_{L}(q)=-e^{2} n\left(E_{\mathrm{F}}\right) \ln \left|\left(q+2 k_{F}\right) /\left(q-2 k_{F}\right)\right|$, которая расходится при $q=2 k_{F}$. Расходимость Линдхарта восприимчивости электронного газа вызвана тем, что волновой вектор $q=2 k_{F}$ связывает сегменты поверхности Ферми с одинаковой энергией, при этом для одномерного газа знаменатель $\chi(q)$ обращается в ноль, а числитель остается конечной величиной. В двух или трехмерном электронном газе поведение $\chi_{L}(q)$ при $q \rightarrow 2 k_{F}$ другое и восприимчивость газа сохраняет конечное значение. Было установлено, что неустойчивость ФП в $\mathrm{La}_{1.2} \mathrm{Sr}_{1.8} \mathrm{Mn}_{2} \mathrm{O}_{7}$ к изменениям $q$ создает зарядовую/орбитальную модуляцию несоразмерную с постоянной кристаллической решетки $a$ с периодом $3.3 a$, которая объединяется с периодическими ян-теллеровскими искажениями кристаллической решетки в виде волны зарядовой/орбитальной плотности. Согласно данным рентгеновского и нейтрон- 
ного рассеяния, орбитальная периодическая структура реально существует в $a b$ плоскостях с длиной когерентности $\sim 20 \AA$. Наличие только короткодействующего ВЗП упорядочения в $\mathrm{La}_{1.2} \mathrm{Sr}_{1.8} \mathrm{Mn}_{2} \mathrm{O}_{7}$ можно было ожидать, так как период пространственной модуляции этой волны несоразмерен с постоянной кристаллической решетки. Такую пространственную модуляцию электронных свойств слоистых манганитов можно рассматривать как наномасштабное разделение фаз в динамическом режиме диэлектрических зарядово-упорядоченных и металлических нанофаз.

В работе [11] в образце $\mathrm{La}_{2-2 x} \mathrm{Sr}_{1+2 x} \mathrm{Mn}_{2} \mathrm{O}_{7}$ с $x=0.38$ при $T=20 \mathrm{~K}$ была экспериментально построена 2D поверхность Ферми. Полученная зависимость энергии связи квазичастиц от величины волнового вектора $k_{x}$ имеет вид параболической дисперсии $E\left(k_{x}\right)$ с широким максимумом спектрального веса вокруг энергии связи $E \sim 0.4 \mathrm{eV}$ относительно $E_{\mathrm{F}}$. Вблизи $E_{\mathrm{F}}$ зависимость энергии связи от $k_{x}$ становится резкой и, повидимому, ренормализована сильной связью дырок с фононами по сравнению с параболической дисперсией характерной для обычных металлов. Результатом этой ренормализации является формирование узкого острого пика спектрального веса в непосредствнной близости от $E_{\mathrm{F}}$, который впервые был обнаружено в образцах $\mathrm{La}_{2-2 x} \mathrm{Sr}_{1+2 x} \mathrm{Mn}_{2} \mathrm{O}_{7}$ с $x=0.36-0.38$ и практически отсутствует в слоистых манганитах с $x \geq 0.4$ с большей анизотропией электронных свойств. В образцах с $x=0.36-0.38$ отсутствуют признаки существования псевдощели в виде широкого провала спектрального веса вблизи $E_{\mathrm{F}}$, что характерно для обычных металлов.

В работе [12] исследовалась природа электронной щели в двухслойных манганитах $\mathrm{La}_{2-2 x} \mathrm{Sr}_{1+2 x} \mathrm{Mn}_{2} \mathrm{O}_{7}$ $(0.3 \leq x \leq 0.5)$ путем изучения спектра оптической проводимости. Было установлено, что особенности зависимости зарядовой щели от $x$ нельзя объяснить только в терминах зарядовых/решеточных корреляций. Предполагается, что необычное поведение щели соответствует кооперативной связи между зарядовыми/орбитальными корреляциями и квазиодномерной зоной вблизи $E_{\mathrm{F}}$. Известно, что зарядовая щель количественно измеряет локализацию носителей в манганитах с решеткой типа перовскита. Стабильность локализации заряда благодаря появлению зарядовых/орбитальных корреляций отражается в величине зарядовой щели. В $\mathrm{La}_{1-x} \mathrm{Ca}_{x} \mathrm{MnO}_{3}$ рост зарядовой щели вблизи $x \approx 0.5$ обычно относится к сильным зарядовым/орбитальным флуктуациям $C E$-типа, возникающим из-за близости к бикритической точке. В $\mathrm{La}_{2-2 x} \mathrm{Sr}_{1+2 x} \mathrm{Mn}_{2} \mathrm{O}_{7}$ рост зарядовой щели вблизи $x=0.4$ нельзя объяснить в рамках этой модели. Связь между зарядовой щелью и устойчивостью зарядовых/орбитальных корреляций в этом соединении напоминает сценарий волны зарядовой плотности. Когда ВЗП уже сформирована, величина щели пропорциональна решеточным смещениям. Интенсивность пика сверхрешетки, возникающего вследствие зарядовой/решеточной модуляции, пропорциональна квадрату решеточного смещения. Это подтверждается результатами работы [12]. Более того, ранее проведенные ARPES измерения показали, что для $x=0.4$ имеется квазиодномерная зонная структура вблизи $E_{\mathrm{F}}$ необходимая для образования ВЗП $[13,10]$. Однако обычная ВЗП модель не может объяснить некоторые результаты: размер найденной щели $\sim 0.3 \mathrm{eV}$ слишком велик для ВЗП щели; края щели пологие, что не характерно для ВЗП; размеры зарядовых/орбитальных корреляций составляют лишь несколько нанометров; с понижением температуры интенсивность рентгеновского и нейтронного рассеяния подавлена. Авторы предлагают новое возможное объяснение аномальной зависимости щели от $x$ - коллективное взаимодействие между локализацией, связанной с образованием зарядовых или орбитальных корреляций, и делокализацией вследствие странствующего движения носителей в $a b$ плоскостях. В то время как зарядовые или решеточные корреляции ослабевают при уменьшении $x$, подвижность носителей заряда возрастает. Спектральный вес квазичастиц с энергией $\leq 0.1 \mathrm{eV}$ при этом также растет, что уменьшает размер щели. Для $x \approx 0.4$ при температурах выше $T_{c}$ короткодействующие или динамические ФМ спиновые корреляции сохраняются, поэтому подвижность носителей заряда не исчезает. При этом, формируется квазиодномерная поверхность Ферми, которая сосуществует с короткодействующей модуляцией зарядовой плотности и кристаллической решетки [10].

Эволюция найденных пиковых особенностей намагниченности $M(T)$ в самодопированных манганитах $\mathrm{La}_{1-y} \mathrm{Sm}_{y} \mathrm{MnO}_{3+\delta}(\delta \sim 0.1, y=0.85,1.0)$ с ростом температуры и напряженности внешнего магнитного поля хорошо согласуется на качественном уровне с приведенными выше результатами комплексного экспериментального исследованиями квазидвумерных манганитов и моделями формирования в них ВЗП/ВСП одночастичных состояний. Однако обнаруженная нами сильная зависимость этих особенностей от относительно слабых внешних воздействий, появление аномального ферромагнетизма и ряд других вопросов выходят за рамки рассмотренных моделей. Поэтому, представляет интерес рассмотреть современные модели появления ВЗП/ВСП состояний и сверхпроводимости, основанные на возможности существовании в некоторых материалах необычного состояния - экситонного конденсата (ЭК), в котором согласно литературным данным могут возникать под влиянием слабых внешних воздействий новые типы волн зарядовой/спиновой плотности и сверхпроводимости, тесно связанные с различыми видами нестинга электрондырочных участков поверхности Ферми и межзонного (внутризонного) спаривания носителей заряда и спина.

В работе [16] была построена двухзонная модель формирования экситонной ВСП с учетом межзонного и внутризонного взаимодействий носителей заряда. Рассмотрен случай полного нестинга электрон-дырочных участков поверхности Ферми при нескольких различных значениях волнового вектора $\mathbf{Q}_{\mathbf{i}}$. Было показано, 
что изменение формы поверхности Ферми, вызванное относительным сдвигом зоны проводимости и валентной зоны, приводит к конкуренции различных магнитных фаз. В рамках этой модели была исследована статическая магнитная восприимчивость электрон-дырочной системы, что позволило определить природу ее различных неустойчивостей типа экситонной ВСП. Показано, что неустойчивости парамагнитного состоянии системы спинов ЭК можно строго идентифицировать, исследуя пиковые особенности температурной зависимости $\chi(T)$ их статической магнитной восприимчивости. Когда с понижением температуры парамагнитного состояния электрон-дырочной системы она достигает критического значения, то можно ожидать появления пиков $\chi(T)$ различной формы, вызванных появлением ВСП с волновыми векторами равными различным значениям вектоpa $\mathbf{Q}_{\mathbf{i}}$ нестинга электрон дырочных участков ФП. Было установлено, что в случае экситонной (межзонной) неустойчивости, особенность парамагнитной восприимчивости вызванная нестингом ФП имеет вид острого пика, тогда как для внутризонной неустойчивости характерна пиковая особенность, размазанная в широком интервале значений волнового вектора вблизи $\mathbf{Q}_{\mathbf{i}}$. Этот результат может быть использован в дальнейшем при более детальном анализе возможных причин найденного нами сильного уширения пик-холм особенности $M(T)$ в $\mathrm{La}_{1-y} \mathrm{Sm}_{y} \mathrm{MnO}_{3+\delta}(y=0.85,1.0)$ с ростом напряженности внешнего магнитного поля.

Особый интерес вызывает обнаруженное нами появление в самодопированных $\mathrm{La}_{0.15} \mathrm{Sm}_{0.85} \mathrm{MnO}_{3+\delta}$ манганитах при температурах ниже $T_{\mathrm{FM}} \approx 12 \mathrm{~K}$ в магнитных полях $H>350$ Ое признаков аномального ферромагнетизма. Ферромагнетизм ЭК впервые был исследован теоретически в работах [17-19]. Была рассмотрена модель спектра фермионов $(S=1 / 2)$, которые нестабильны к электрон-дырочному спариванию в пределе слабой связи. Найдены условия при которых синглетное $(S=0)$ и триплетное $(S=1)$ виды спаривания спинов могут сосуществовать, что приводит к необычному состоянию газа фермионов типа экситонного феромагнетика. Авторы рассмотрели три вида газа фермионов; 1) полуметаллы с перекрывающимися ФП, 2) полупроводники с узкой запрещенной зоной, меньшей чем энергия связи экситона, 3) металлы с очень узкими зонами. Во всех трех случаях система неустойчива к когерентному электрон-дырочному спариванию свободных носителей заряда в синглетное состояние с энергией связи $V_{\mathrm{C}}$ или триплетное состояние с энергией связи $V_{\mathrm{T}}$, или к образованию двух состояний, которые отличаются векторами q. Энергетический спектр таких систем имеет полупроводниковый характер с диэлектрической щелью $\Delta_{\mathrm{C}}$ при синглетном спаривании и $\Delta_{\mathrm{T}}$ при триплетном. Было показано, что переход газа свободных носителей заряда в таких материалах в ферромагнитное основное состояние возможен даже в случае слабого взаимодействия между носителями заряда. Установлено, что одновременное существование синглетного $\left(\Delta_{\mathrm{S}}\right)$ и триплетного $\left(\Delta_{t}\right)$ параметров порядка сопровождается образованием ВЗП и ВСП, а также снятием вырождения по спину электронной и дырочной зон. В этом случае, если число электронов не равно числу дырок, число носителей заряда с противоположно направленным спином различно. Полный спин такой системы пропорционален разности концентраций электронов и дырок, что является причиной появления экситонного ферромагнетизма при $T \rightarrow 0$. Было получено, что при температурах выше перехода спаренных носителей заряда в ферромагнитное сосостояние температурная зависимость статической магнитной восприимчивости описывается законом Кюри. В более поздней обзорной работе [20] было отмечено, что приближение слабой связи, в котором отсутствует хундовская сильная связь, хорошо описывает слабо коррелированные металлы или полупроводники, в которых склеивание электрон-дырочных пар осуществляется дальнодействующей частью взаимодействия. Это приводит к сосуществованию в этих материалах множества вырожденных экситонных состояний. Благодаря этому вырождению даже такое слабое обменное взаимодействие как перескоки электрон-дырочных пар между атомами может играть важную роль при формировании экситонного ферромагнетизма и ВЗП/ВСП упорядоченных состояний фермионов [21,22]. Согласно [21] в ЭК могут сосуществовать волна зарядовой плотности с синглетным спариванием спинов $(S=0)$ и волна спиновой плотности с триплетным спариванием спинов $(S=1)$. В моделях слабой связи предполагается, что основной причиной ЭК-неустойчивости является полный нестинг электрон-дырочных участков поверхности Ферми. По нашему мнению, рассмотренные выше модели неустойчивости экситонного конденсата к внешним воздействиям в простейшем случае предела слабой связи могут быть использованы в дальнейшем исследовании.

\section{4. Заключение}

Мы считаем, что обнаруженные в $\mathrm{La}_{0.15} \mathrm{Sm}_{0.85} \mathrm{MnO}_{3+\delta}$ в слабом поле $H=100$ Ое два острых пика $M(T)$ различной интенсивности при близких температурах $T_{1}$ и $T_{2}$ чуть выше критической температуры $T_{c}$ фазового перехода в когерентное состояние с локальной ВТСП соответствуют расходимости Линдхарда температурной зависимости магнитной восприимчивости $1 \mathrm{D}$ газа квазичастищ $\chi_{L}\left(q_{\text {nest }}\right)$ при значениях волнового вектора $q_{\text {nest } 1}=2 k_{F 1}$ в и $q_{\text {nest } 2}=2 k_{F 2}$ в двух пространственно разделенных областях образца (условно „металлическая“ и „диэлектрическая“ фаза) с большой и малой плотностью состояний свободных носителей заряда/спина на уровне Ферми $N_{1}\left(E_{\mathrm{F}}\right) \ll N_{2}\left(E_{\mathrm{F}}\right)$. В пределе слабой связи, появление таких областей можно объяснить нестингом электрон-дырочных участков поверхности Ферми, что сопровождается появлением в $a b$ плоскостях при температурах $T<60 \mathrm{~K}$ пространственной модуляции электронных и магнитных свойств 
в виде наномасштабных фрагментов флуктуирующих квазиодномерных волн зарядовой/спиновой плотности несоразмерных с кристаллической решеткой с волновыми векторами $q_{1} \| a$ и $q_{2} \| b$ направлениям. С ростом напряженности магнитного поля до критического значения $H=1050$ Ое происходит сильное уширение и выравнивание интенсивностей пиков намагниченности вблизи температур $T_{1}$ и $T_{2}$, что объясняется в работе уменьшением псевдощели $\Delta$ в спектре носителей заряда с ростом $H$, которое сопровождается изменением плотности их состояний $N_{1}\left(E_{\mathrm{F}}\right)$ и $N_{2}\left(E_{\mathrm{F}}\right)$ в двух областях. В полях $H>1050$ Ое происходит почти полное подавление магнитного отклика $\chi_{L}\left(q_{\text {nest }}\right)$ с $q_{1} \| a$ вблизи $T_{1}$, которое сопровождается формированием при $T<T_{\mathrm{FM}}=12.5 \mathrm{~K}$ „металлической“ ФМ микрофазы. Рост напряженности $H$ внешнего магнитного поля приводит к росту плотности состояний свободных носителей заряда/спина на $E_{\mathrm{F}}$ и трансформации ВЗП/ВСП корреляций в $1 \mathrm{D}$ ВСП состояние. Предполагается, что обнаруженное в данной работе сильное влияние относительно слабых внешних воздействий (изменение состава, напряженности внешнего магнитного поля) на намагниченность и сверхпроводимость в фрустрированных манганитах $\mathrm{La}_{1-y} \mathrm{Sm}_{y} \mathrm{MnO}_{3+\delta}(y=0.85,1.0)$ объясняется кроссовером основных состояний щелевой спиновой и сверхпроводящей квантовых жидкостей в этих образцах, что приводит к появлению сильных флуктуаций соответствующих локальных параметров порядка вблизи квантовой критической точки. Эволюция найденных пиковых особенностей намагниченности $M(T)$ в самодопированных манганитах $\mathrm{La}_{1-y} \mathrm{Sm}_{y} \mathrm{MnO}_{3+\delta}(\delta \sim 0.1, y=0.85,1.0)$ с ростом температуры и напряженности внешнего магнитного поля хорошо согласуется на качественном уровне с результатами комплексного экспериментального исследованиями квазидвумерных манганитов и моделями формирования в них флуктуирующих фрагментов 1D ВЗП/ВСП состояний в приближении слабой связи между квазичастицами.

\section{Конфликт интересов}

Авторы заявляют, что у них нет конфликта интересов.

\section{Список литературы}

[1] V.J. Emery, S.A. Kivelson. Physica C 209, 597 (1993).

[2] V.J. Emery, S.A. Kivelson, O. Zachar. Phys. Rev. B 56, 6120 (1997).

[3] S.A. Kivelson, E. Fradkin, V.J. Emery. Nature 393, 550 (1998).

[4] V.J. Emery, S.A. Kivelson, J.M. Tranquada. Proc. Natl. Acad. Sci. (USA) 96, 8814 (1999).

[5] V.J. Emery, S.A. Kivelson, J.M. Tranquada. PNAS 96, 8814 (1999).

[6] S.A. Kivelson, I.P. Bindloss, E. Fradkin, V. Oganesyan, J.M. Tranquada, A. Kapitulnik, C. Howald. Rev. Mod. Phys. 75, 1201 (2003).

[7] Ф.Н. Буханько, А.Ф. Буханько. ФТТ 57, 1098 (2015).

[8] Ф.Н. Буханько, А.Ф. Буханько. ФТТ 58, 506 (2016).
[9] D.S. Dessau, T. Saitoh, C.-H. Dark, Z.-X. Shen, P. Villella, N. Hamada, Y. Moritomo, Y. Tokura. Phys. Rev. Lett. 81, 192 (1998); T. Saitoh, D.S. Dessau, Y. Moritomo, T. Kimura, Y. Tokura, N. Hamada. Phys. Rev. B 62, 1039 (2000).

[10] Y.-D. Chuang, A.D. Gromko, D.S. Dessau, T. Kimura, Y. Tokura. Science 292, 1509 (2001)

[11] Z. Sun, Y.D. Chuang, A.V. Fedorov, J.F. Douglas, D. Reznik, F. Weber, N. Aliouane, D.N. Argyrion, H. Zheng, J.F. Mitchell, T. Kimura, Y. Tokura, A. Revcolevschi. D.S. Dessau. Phys. Rev. Lett. 97, 055401 (2006); Z. Sun, J.F. Douglas, Q. Wang, D.S. Dessau, A.V. Fedorov, H. Lin, S. Sahrakorpi, B. Barbiellini, R.S. Markiewicz, A. Bansil, H. Zheng, J.F. Mitchell. Phys. Rev. B 78, 075101 (2008).

[12] Myung Whun Kim, H.J. Lee, B.J. Yang, K.H. Kim, Y. Moritomo, Jaejun Yu, T.W. Noh. Phys. Rev. Lett. 98, 187201 (2007).

[13] N. Mannella, W.L. Yang, X.J. Zhou, H. Zheng, J.F. Mitchell, J. Zaanen, T.P. Devereaux, N. Nagaosa, Z. Hussain, Z.-X. Shen. Nature (London) 438, 474 (2005).

[14] R. Saniz, M.R. Norman, A.J. Freeman. Phys. Rev. Lett. 101, 236402 (2008).

[15] J. Salafranca, G. Alvarez, E. Dagotto. Phys. Rev. B 80, 155133 (2010).

[16] Björn Zocher, Carsten Timm, P.M.R. Brydon. Phys. Rev. B 84, 144425 (2011).

[17] B.A. Volkov, Y.V. Kopaev. JETP Lett. 19, 104 (1973).

[18] B.A. Volkov, Y.V. Kopaev, A.I. Rusinov. Sov. Phys. JETP 41, 952 (1975).

[19] B.A. Volkov, A.I. Rusinov, R.K. Timerov. Sov. Phys. JETP 43, 589 (1976).

[20] J. Kuneš. J. Phys.: Condens. Matter 27, 333201 (2015).

[21] B.I. Halperin, T.M. Rice. Solid State Physics 21, 115 (1968).

[22] B.I. Halperin, T.M. Rice. Rev. Mod. Phys. 40, 755 (1968).

Редактор Т.Н. Василевская 